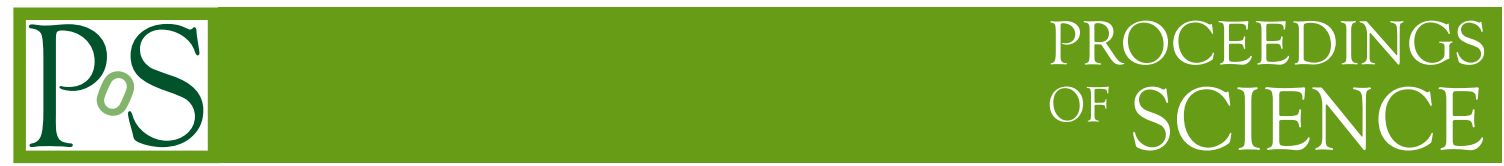

\title{
Nuclear astrophysics deep underground: the LUNA experiment
}

\author{
Chiara Mazzocchi for the LUNA collaboration* \\ Università degli Studi di Milano and INFN, Sez. Milano, 20133 Milan, Italy \\ E-mail: chiara.mazzocchi@mi.infn.it
}

\begin{abstract}
Measuring nuclear reactions of astrophysical interest at the relevant energies is not always possible on the Earth's surface because of the cosmic-ray background that dominates the spectra. The LUNA collaboration exploits the low-background environment of Gran Sasso National Laboratory to study these reactions at or close to the Gamow peak. The status is summarized in this contribution.
\end{abstract}

XLVIII International Winter Meeting on Nuclear Physics, BORMIO2010

January 25-29, 2010

Bormio, Italy

${ }^{*}$ Speaker. 


\section{Introduction}

Mankind has been trying to answer one fundamental question for almost as long as it exists: how do the Sun and the stars shine? It is now well known that these celestial objects are powered by nuclear reactions that generate energy and produce the elements in the Universe. Big Bang nucleosynthesis produced no isotopes heavier than ${ }^{7} \mathrm{Li}$. Stars like our Sun "shine" because they generate energy over billions of years by thermonuclear fusion of hydrogen to helium ("hydrogen burning"). Once the hydrogen is exhausted, the next burning stage is ignited and fuses ${ }^{4} \mathrm{He}$ into ${ }^{12} \mathrm{C}$ and ${ }^{16} \mathrm{O}$ ("helium burning"). The subsequent advanced burning stages (carbon, neon, oxygen, and silicon burning) build up elements up into the iron region and proceed only in stars 8 times more massive than our Sun. In this paper only the hydrogen burning processes in the pp-chain and the $\mathrm{CNO}$ and $\mathrm{MgAl}$ cycles will be addressed.

Many models have been developed in order to describe stellar systems. In recent years, due to the resources provided by the new high performance computers, the models can describe the stellar behavior also in three dimensions extending our knowledge of the stellar interior. One critical input of these models is the cross section of the nuclear reactions. The knowledge of the cross sections at stellar energies is a key issue in the field of nuclear astrophysics, with the ambitious task to explain the origin and observed relative abundances of the elements in the Universe.

Inside a star non-resonant nuclear-fusion reactions induced by charged particles take place within a narrow energy window, the so-called Gamow peak. Typical energies for the Gamow peak of hydrogen-burning reactions are few to tens of $\mathrm{keV}$. These are much lower than the respective Coulomb barrier, which is typically $0.5-2 \mathrm{MeV}$. At such low energies, the non-resonant reactions cross-section $\sigma(\mathrm{E})$ drops almost exponentially with decreasing energy, because of the tunneling probability through the Coulomb barrier. The cross section can be then written as

$$
\sigma(E)=\frac{S(E)}{E} e^{-2 \pi \eta}
$$

where $\mathrm{E}$ is the energy in the centre of mass reference system, $\eta(\mathrm{E})$ is the Sommerfeld parameter with $2 \pi \eta=31.29 \mathrm{Z}_{p} \mathrm{Z}_{t} \sqrt{\mu / E}, \mathrm{Z}_{p}$ and $\mathrm{Z}_{t}$ are the charge numbers of the projectile and target nucleus, respectively, $\mu$ the reduced mass in amu. $\mathrm{S}(\mathrm{E})$ is the astrophysical S-factor, which contains all the strictly nuclear effects, while the exponential drop due to the Coulomb barrier is taken into account by the exponential term.

At such low energies as those relevant for astrophysics, the reaction cross sections are very small, often of the order of pbarn to nbarn and it becomes increasingly difficult with decreasing energy to measure them directly in the laboratory. In fact the conditions for measuring these cross sections in the laboratory (with typical beam currents of the order of 100 to $500 \mu \mathrm{A}$, target thicknesses of a few $\mu \mathrm{g} / \mathrm{cm}^{2}$ and detection efficiencies of the order of $10 \%$ ) are such that often only a few events/day to a few events/hour can be detected. It is therefore not always possible to push the observation limits to the energy range of direct astrophysical interest. The method commonly used to estimate the cross section, from which the reaction rate is afterwards obtained, is usually an extrapolation to the energies of interest. Unfortunately, an extrapolation is not always a reliable tool and can be rather misleading, as illustrated in Figure 1. The presence of unknown narrow resonances or wide tails of sub-threshold resonances in the extrapolated region could lead to wrong conclusions if not considered properly. One way to overcome this problem is to perform the direct measurement of 
the reaction cross section where the background, mostly due to cosmic rays, can be suppressed effectively. Such a shielding from the cosmic radiation can be offered by a mountain, as is the case of the Gran Sasso National Laboratory (LNGS), which is placed deep underground under the Gran Sasso mountain in central Italy. The underground laboratory is shielded by $\sim 1400 \mathrm{~m}$ of rock overburden (4000 m water equivalent), which suppresses the muon flux by six and the neutron flux by three orders of magnitude with respect to the Earth's surface.

LUNA (Laboratory for Underground Nuclear Astrophysics) exploits the low-background characteristics of LNGS underground location for measuring directly nuclear reactions of astrophysical interest at or close to the Gamow peak.

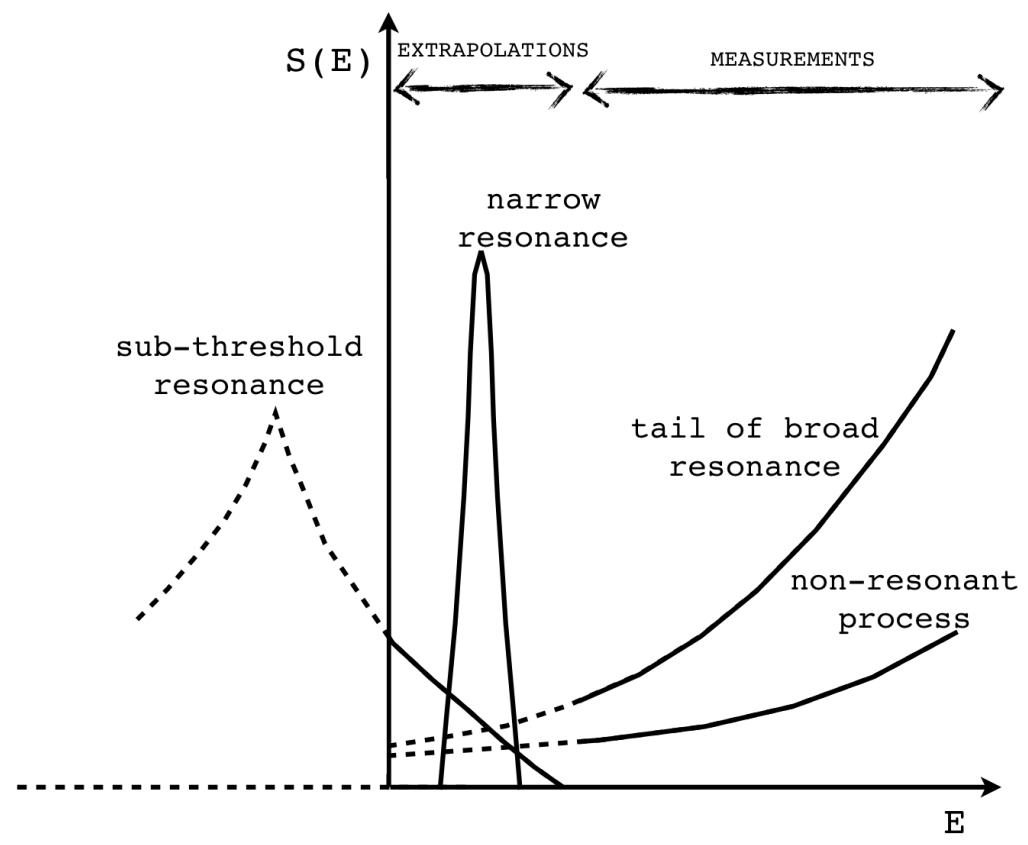

Figure 1: Schematic representation of the astrophysical S-factor as a function of the energy. The dashed lines correspond to the extrapolated trend at energies below the lowest measured one.

\section{The LUNA project}

LUNA started its activity in 1991 by installing a $50 \mathrm{kV}$ electrostatic accelerator underground at LNGS, followed in the year 2000 by a $400 \mathrm{kV}$ machine. The qualifying features of both accelerators are a very small beam energy spread and a very high beam current even at low energies.

The first thermonuclear reactions investigated at LUNA- $50 \mathrm{kV}$ were hydrogen-burning reactions belonging to the pp-chain, namely the $\mathrm{d}(\mathrm{p}, \gamma)^{3} \mathrm{He}[1]$, the ${ }^{3} \mathrm{He}\left({ }^{3} \mathrm{He}, 2 \mathrm{p}\right){ }^{4} \mathrm{He}[2]$ and the $\mathrm{d}\left({ }^{3} \mathrm{He}, 2 \mathrm{p}\right){ }^{4} \mathrm{He}$ [3]. With the LUNA-400 $\mathrm{kV}$ facility it became possible to investigate also reactions like ${ }^{3} \mathrm{He}\left({ }^{4} \mathrm{He}, \gamma\right){ }^{7} \mathrm{Be}$ [4]. In this latter phase, also hydrogen-burning reactions belonging to the $\mathrm{CNO}$ and $\mathrm{MgAl}$ cycles, as well as reactions for Big-Bang nucleosynthesis could be investigated.

Hydrogen-burning in the CNO cycle: ${ }^{15} \mathbf{N}(\mathbf{p}, \gamma){ }^{16} \mathrm{O}$. The ${ }^{15} \mathrm{~N}(\mathrm{p}, \gamma){ }^{16} \mathrm{O}$ reaction is an important reaction of the $\mathrm{CNO}$ cycles. As a matter of fact it links the first $\mathrm{CNO}$ cycle to the second one (see 
Figure 2) allowing for the production of the oxygen and fluorine isotopes and giving the access also to the third and fourth cycles that are responsible of the production of elements until neon.

The ${ }^{15} \mathrm{~N}$ can interact with protons via $(\mathrm{p}, \gamma)$ and $(\mathrm{p}, \alpha)$ reactions. The branching ratio, or the ratio of probabilities for the occurrence of the $(\mathrm{p}, \alpha)$ (strong nuclear interaction) and (p, $\gamma$ ) (weaker electromagnetic interaction) reaction, is given by the ratio of the corresponding reaction rates. In the case of ${ }^{15} \mathrm{~N}(\mathrm{p}, \gamma){ }^{16} \mathrm{O}$, this ratio determines after how many cycles of CNO I the carbon will be lost as catalyst and the CNO II will proceed. Varying the S-Factor of ${ }^{15} \mathrm{~N}(\mathrm{p}, \gamma){ }^{16} \mathrm{O}$ by a factor two could change the amount of ${ }^{16} \mathrm{O}$ production by $30 \%$ [5].

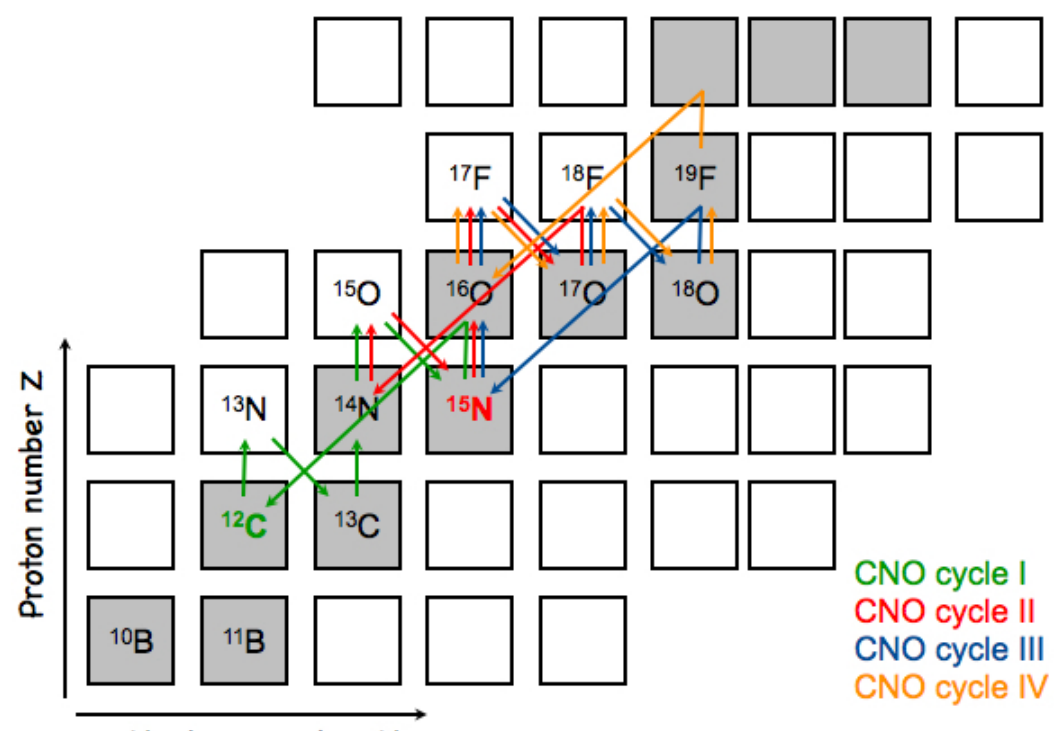

Neutron number $\mathrm{N}$

Figure 2: Portion of the chert of nuclei with reaction paths of the four $\mathrm{CNO}$ cycles.

Hydrogen-burning in the MgAl cycle: ${ }^{25} \mathrm{Mg}(\mathbf{p}, \gamma){ }^{26} \mathrm{Al} . \quad \mathrm{The}{ }^{25} \mathrm{Mg}(\mathrm{p}, \gamma){ }^{26} \mathrm{Al}$ reaction is the slowest of the Mg-Al cycle (see Figure 3$)$. The $\beta^{+}$decay of ${ }^{26} \mathrm{Al}\left(\mathrm{T}_{1 / 2}=7 \cdot 10^{5} \mathrm{y}\right)$ populates the 1.8 $\mathrm{MeV}$ excited state of ${ }^{26} \mathrm{Mg}$ which then decays to the ground state by $\gamma$-emission (see Figure 3 ). Observations from satellites $[6,7]$ have determined an amount of ${ }^{26} \mathrm{Al}$ in our galaxy of about 6 solar masses through the detection of this $\gamma$-ray line. Moreover, the presence of ${ }^{26} \mathrm{Al}$ in the interstellar medium has been determined from the observation of ${ }^{26} \mathrm{Mg}$ isotopic enrichment (extinct ${ }^{26} \mathrm{Al}$ ) in carbonaceous meteorites [8]. While the former observation gives evidence that ${ }^{26} \mathrm{Al} \mathrm{nu}$ cleosynthesis is still active on a large scale, the latter suggests that ${ }^{26} \mathrm{Al}$ was produced not later than 4.6 billion years ago (time of the condensation of the solar-system material). ${ }^{26} \mathrm{Al}$ is mainly produced via the ${ }^{25} \mathrm{Mg}(\mathrm{p}, \gamma){ }^{26} \mathrm{Al}$ reaction which is active in the hydrogen burning shell of off-main sequence stars of any mass and in the carbon burning regions of massive stars. Therefore different astrophysical environments are responsible for ${ }^{26} \mathrm{Al}$ nucleosynthesis and the reaction cross section should be precisely studied in a wide energy range since each burning stage is characterized by a different temperature range corresponding to a different Gamow window.

Big-bang nucleosynthesis: ${ }^{2} \mathbf{H}\left({ }^{4} \mathbf{H e}, \gamma\right){ }^{6} \mathbf{L i}$. Recently, the ${ }^{6} \mathrm{Li}$ isotope has been detected in a num- 


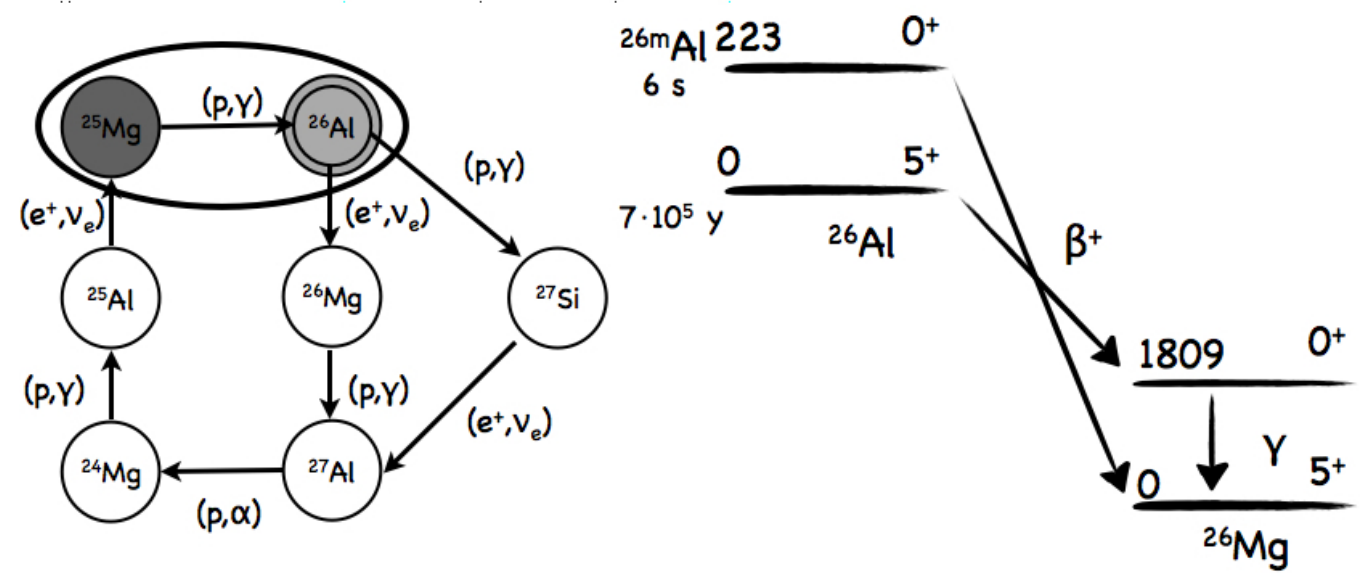

Figure 3: Left panel: reaction path followed by the $\mathrm{MgAl}$ cycle through ${ }^{26 g . s .} \mathrm{Al}$ and ${ }^{26 m} \mathrm{Al}$. Right panel: decay scheme of ${ }^{26} \mathrm{Al}$.

ber of metal-poor stars $[9,10]$. These observations are significant because they suggest a ${ }^{6} \mathrm{Li}$ plateau similar to the well-known Spite plateau for ${ }^{7} \mathrm{Li}[11]$. The observed ${ }^{7} \mathrm{Li}$ abundance (e.g. [12] and [10]) is a factor of 3 below the value predicted by standard Big-Bang nucleosynthesis based on the baryon-to-photon ratio from cosmic microwave background observations [13]. The ${ }^{6} \mathrm{Li}$ data, however, are higher by as much as two to three orders of magnitude than the predicted Big-Bang production. This gives rise to a ${ }^{6} \mathrm{Li}$ problem in Big-Bang nucleosynthesis, in addition to the well-known ${ }^{7} \mathrm{Li}$ problem. If the existence of the ${ }^{6} \mathrm{Li}$ plateau is confirmed, a pre-galactic source of ${ }^{6} \mathrm{Li}$ has to be introduced as an explanation. This could either be an extremely enhanced production in Big-Bang nucleosynthesis, mainly through the ${ }^{2} \mathrm{H}\left({ }^{4} \mathrm{He}, \gamma\right)^{6} \mathrm{Li}$ reaction (see Figure 4), or nonstandard physics like the decay of relic gravitinos [14]. It has been shown that ${ }^{6} \mathrm{Li}$ production through the pre-galactic interaction of energetic particles with the interstellar medium cannot explain the level of detected ${ }^{6} \mathrm{Li}$ [15]. Most scenarios of pre-galactic ${ }^{6} \mathrm{Li}$ production, except for an enhanced ${ }^{2} \mathrm{H}\left({ }^{4} \mathrm{He}, \gamma\right){ }^{6} \mathrm{Li}$ cross-section, produce not only ${ }^{6} \mathrm{Li}$ but also ${ }^{7} \mathrm{Li}$, therefore worsening the ${ }^{7} \mathrm{Li}$ problem. A sensitivity study of nuclear reaction rates for Big-Bang nucleosynthesis has shown that the nuclear uncertainty in the predicted ${ }^{6} \mathrm{Li}$ abundance is dominated by the uncertainty in the ${ }^{2} \mathrm{H}\left({ }^{4} \mathrm{He}, \gamma\right){ }^{6} \mathrm{Li}$ cross-section [16].

\section{The experiments}

The LUNA-400 kV facility consists of an accelerator with two beam lines. Intense beams of protons or alpha particles are accelerated up to $400 \mathrm{keV}$ with intensities up to 500 and $200 \mu \mathrm{A}$, respectively. The beam lines are equipped with different experimental set-ups. Both solid-state or (windowless) gas targets can be used, according to the reaction of interest. For $\gamma$-ray detection a high-efficiency low-resolution BGO and a high-resolution low-efficiency HPGe detector are available.

Among the reactions described in the previous section the ${ }^{15} \mathrm{~N}(\mathrm{p}, \gamma){ }^{16} \mathrm{O}$ reaction has been extensively studied using the BGO detector with both the isotopically enriched solid-state and the win- 


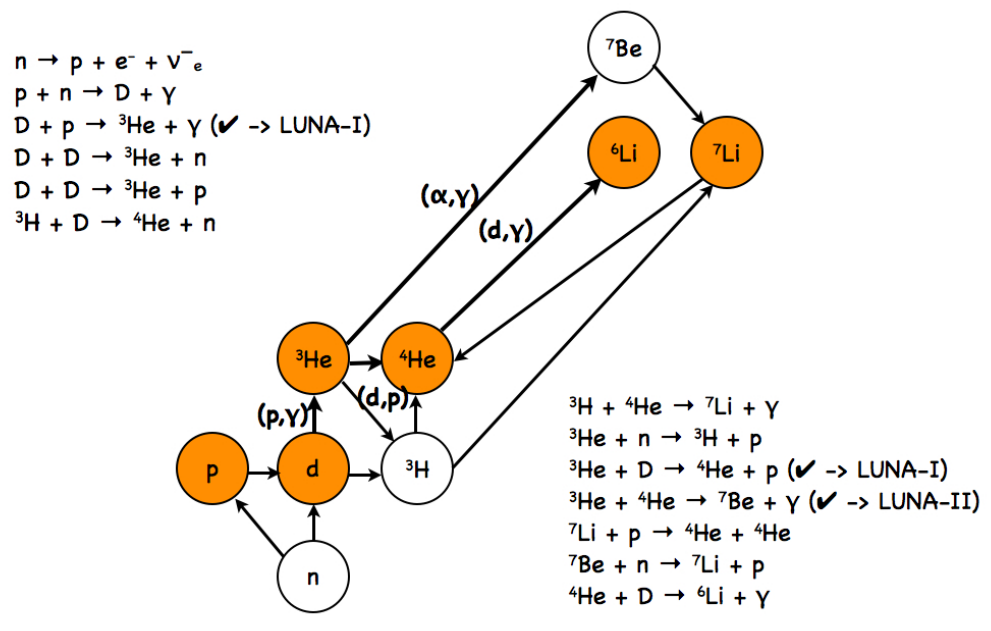

Figure 4: Reactions belonging to the Big-Bang nucleosynthesis.

dowless gas target approach. The results of the latter measurement have been recently published [17], while those of the former will be published soon. In an effort to study the same reaction over a wider energy range, another measurement was performed at LUNA with an HPGe detector and solid-state isotopically enriched targets in collaboration with the University of Notre Dame, USA. The same set-up was used at three different accelerators (LUNA, covering the lower energy range, and two machines at the University of Notre Dame, covering the higher energies) to span over a wide energy interval (100-2000 keV) in the same experimental conditions. Data analysis is in progress. It will allow to perform R-matrix fits with a unique set of data and therefore study the energy dependence of the astrophysical S-factor.

As far as the ${ }^{25} \operatorname{Mg}(\mathrm{p}, \gamma)^{26} \mathrm{Al}$ reaction is concerned, resonances at 93, 130, 190 and $304 \mathrm{keV}$ were investigated using different experimental approaches, both natural and enriched Mg targets. The 304 $\mathrm{keV}$ resonance was measured at LUNA with three different techniques (HPGe, BGO and AMS), a very good agreement was found among the different approaches for the resonance strength [18]. By comparing the LUNA result with literature data, a strong disagreement is found only with the result of Arazi et al. [19] obtained with the AMS (Accelerator Mass Spectrometry) technique for the $190 \mathrm{keV}$ resonance. The evaluation of NACRE [20] combined with the LUNA result yields an improved uncertainty of 4\%. Among the other resonances, the $190 \mathrm{keV}$ resonance was studied with both HPGe and BGO approaches, while the two at lower energies could be investigated only by means of the BGO detector, thanks to its high-efficiency. In all three cases the data analysis is on-going.

The last of the reactions quoted in the previous section, ${ }^{2} \mathrm{H}\left({ }^{4} \mathrm{He}, \gamma\right){ }^{6} \mathrm{Li}$, is extremely challenging to measure. Its low-energy cross-section has never been measured directly in the region of interest $\left(0.05<\mathrm{E}_{c m}<0.5 \mathrm{MeV}\right)$. The cross-section of the ${ }^{2} \mathrm{H}\left({ }^{4} \mathrm{He}, \gamma\right){ }^{6} \mathrm{Li}$ reaction was measured in the energy range of Big-Bang nucleosynthesis in a Coulomb dissociation study at Karlsruhe [21]. Theoretical 
calculations [22] yield values that are systematically lower than the data. The NACRE compilation [20] consequently cites an uncertainty of a factor 3 in the rate at Big-Bang nucleosynthesis temperatures. It is therefore important to measure this reaction directly at the energies of interest. This measurement can be performed at LUNA using an $\alpha$-beam, a deuterium gas-target and a HPGe detector (135\% efficiency) mounted in close geometry. Tests on the set-up are ongoing.

\section{Outlook}

The program for the near future includes the study of the above mentioned ${ }^{2} \mathrm{H}\left({ }^{4} \mathrm{He}, \gamma\right)^{6} \mathrm{Li}$ reaction. Moreover, proton-capture reaction studies on ${ }^{17} \mathrm{O}$ and ${ }^{18} \mathrm{O}$ are foreseen as well as on ${ }^{22} \mathrm{Ne}$, the latter belonging to the NeNa cycle. In addiction, the LUNA collaboration prepared a Letter of Intent (LOI) with the ambitious program of installing a $3 \mathrm{MV}$ machine at LNGS. This could allow the study of helium-burning reactions among which the ${ }^{12} \mathrm{C}(\alpha, \gamma){ }^{16} \mathrm{O}$ "holy grail" of nuclear astrophysics and of several $(\alpha, \gamma)$ and $(\mathrm{p}, \gamma)$ processes having deep consequences in nucleosynthesis, stellar evolution, supernova mechanism, etc. The proposed LOI received very positive response but the decision of LNGS is still pending, major problems being space requirements and possible interferences with other low-background experiments.

\section{References}

[1] C. Casella et al., Nucl. Phys. A 706 (2002) 203.

[2] R. Bonetti et al., Phys. Rev. Lett. 82 (1999) 5205.

[3] H. Costantini et al., Phys. Lett. B 482 (2000) 43.

[4] D. Bemmerer et al., Phys. Rev. lett. 97 (2006) 122502.

[5] C.Iliadis et al., Ap. J. Supp. Series 142 (2002) 105.

[6] J. Knodlseder et al., Astron, Astroph 345 (1999) 813.

[7] C. Winkler et al Astron. Astroph. 411 (2003) L1.

[8] G. J. Wasserburg in Protostars and Planets II, ed. by D.C. Black and M.S. Matthews (1985) 703.

[9] V. Smith et al., Astrophys. J. 408 (1993) 262.

[10] M. Asplund et al., Astrophys. J. 644 (2006) 229.

[11] F. Spite and M. Spite, Astron. Astrophys. 115 (1982) 357.

[12] P. Bonifacio et al., Astron. Astrophys. 390 (2002) 91.

[13] A. Coc et al., Ap. J. 600 (2004) 544.

[14] K. Jedamzik, Phys. Rev. D 74 (2006) 10309.

[15] N. Prantzos, Astron. Astrophys. 448 (2006) 665.

[16] E. Vangioni-Flam et al., Astron. Astrophys. 360 (2000) 15.

[17] D. Bemmerer et al, J. Phys. G. 36 (2009) 045202.

[18] B.N. Limata et al., submitted to Phys Rev C. 
[19] A. Arazi et al., Phys. Rev. C 74 (2006) 025802.

[20] C. Angulo et al., Nucl. Phys A 656 (1999) 3.

[21] J. Kiener et al., Phys. Rev. C 44(1991) 2195.

[22] A. Kharbach and P. Descouvemont, Phys. Rev. C 58 (1998) 1066. 